\title{
Molybdenum Disilicide - Diffusion, Defects, Diffusion Correlation, and Creep
}

\author{
Helmut Mehrer ${ }^{1, a}$, Hans-Eckhardt Schaefer, ${ }^{2, b}$, \\ Irina V. Belova ${ }^{3, \mathrm{c}}$, and Graeme E. Murch ${ }^{3, \mathrm{~d}}$ \\ ${ }^{1}$ Institut für Materialphysik, Universität Münster \\ Wilhelm-Klemm Str.10, D-48149 Münster, Germany \\ Private address: D-73635 Obersteinenberg, Berghof, Germany \\ ${ }^{2}$ Institut für Theoretische und Angewandte Physik, Universität Stuttgart \\ Pfaffenwaldring 57, D-70569 Stuttgart, Germany \\ ${ }^{3}$ Centre for Mass and Thermal Transport in Engineering Materials, School of Engineering, The \\ University of Newcastle, Callaghan, NSW 2308 Australia \\ a mehrer@uni-muenster.de, ${ }^{b}$ hans-eckhardt.schaefer@itap.uni-stuttgart.de \\ Irina.Belova@newcastle.edu.au, 'Graeme.Murch@newcastle.edu.au
}

\begin{abstract}
Keywords: Molybdenum disilicide, tracer diffusion, vacancies, positron annihilation, jump rates, correlation factors, anisotropy ratios, sublattice vacancy mechanism, Monte Carlo simulations, diffusional creep, dislocation creep
\end{abstract}

\begin{abstract}
Molybdenum disilicide $\left(\mathrm{MoSi}_{2}\right)$ is an interesting material for high-temperature applications. It has a high melting temperature, good thermal and electrical conductivity and an excellent oxidation resistance. For many years the primary use of $\mathrm{MoSi}_{2}$ has been in heating elements, which can be used for temperatures up to $1800^{\circ} \mathrm{C}$. Since the 1990 s the potential of $\mathrm{MoSi}_{2}$ as a high-temperature structural material has been recognized as well. Its brittleness at lower temperatures and a poor creep resistance above $1200^{\circ} \mathrm{C}$ have hindered its use as in load-bearing parts. These disadvantages may be offset at least partly by using it together with a second material in a composite or an alloy. Projected applications of $\mathrm{MoSi}_{2}$-based materials include, e.g. stationary hot section components in gas turbine engines and glow plugs in diesel engines.

For future research and development directions of $\mathrm{MoSi}_{2}$-based composites diffusion is a crucial property because creep is closely connected with diffusion. This paper is devoted to the basic diffusion and defect properties of $\mathrm{MoSi}_{2}$. Data of $\mathrm{Si}$ and Mo as well as Ge diffusion from the Münster laboratory for both principal directions are briefly summarized. For all three kinds of atoms diffusion perpendicular to the tetragonal axis is faster than parallel to it. The diffusivities of Mo in both directions are many orders of magnitude slower than those of Si and Ge. The huge asymmetry between Mo and $\mathrm{Si}$ (or Ge) diffusion suggests that atomic motion of each constituent is restricted to its own sublattice. Positron annihilation studies on $\mathrm{MoSi}_{2}$ from the Stuttgart laboratory are reviewed as well. They show that formation of thermal vacancies occurs primarily on the $\mathrm{Si}$ sublattice but cannot exclude vacancy formation on the Mo sublattice at higher temperatures. Correlation factors for $\mathrm{Si}$ and Mo diffusion via sublattice vacancies in the respective sublattices of $\mathrm{MoSi}_{2}$ have been calculated recently mainly by Monte Carlo simulation techniques and are also briefly described. Diffusion, in particular self-diffusion, is discussed in connection with literature data on high-temperature creep, which is diffusion-controlled. Grain-size effects of creep have been reported and can be attributed to Nabarro-Herring and Coble creep. Power-law creep is attributed to diffusion-controlled dislocation creep. Some details are, however, not completely understood, presumably due to a lack of theoretical concepts for creep in uniaxial, stochiometric compounds and due to missing information on grain-boundary diffusion.
\end{abstract}




\section{Introduction}

Molybdenum disilicide $\left(\mathrm{MoSi}_{2}\right)$ is an intermetallic compound with tetragonal $\mathrm{C} 11_{\mathrm{b}}$ structure formed between molybdenum and silicon. It is a grey metallic-looking material with a high melting point $\left(2030^{\circ} \mathrm{C}\right)$, moderate density $\left(6.24 \mathrm{~g} / \mathrm{cm}^{3}\right)$, good thermal conductivity, and it is electrically conductive. $\mathrm{MoSi}_{2}$ is known since 1907 [1], when it was considered a high-temperature protective material for ductile metals. At high temperatures it forms a thin, coherent, adhesive passivation layer of vitreous silica $\left(\mathrm{SiO}_{2}\right)$ that protects it from further oxidation and provides superb oxidation resistance.

For many years the primary use of $\mathrm{MoSi}_{2}$ has been in heating elements, which can be used for temperatures up to $1800^{\circ} \mathrm{C}$ in electric furnaces in laboratory and production environments. The first commercial heating elements were developed and patented by Kanthal in 1953 [2]. In addition, the good electrical conductivity of $\mathrm{MoSi}_{2}$ in combination with high thermal stability, high electromigration resistance, and excellent diffusion barrier properties are important in various microelectronic applications. Starting in the early 1990s, the potential of $\mathrm{MoSi}_{2}$ as structural material has been recognized. It is brittle at lower temperatures and subject to 'pest oxidation' when exposed to oxidising environments between 400 and $600^{\circ} \mathrm{C}$. The cause of pest effects seems to be grain-boundary diffusion of oxygen. $\mathrm{MoSi}_{2}$ has a brittle-to-ductile transition around $1000^{\circ} \mathrm{C}$. Above this temperature its strength is governed by plastic flow (creep). It has a poor creep resistance above $1200^{\circ} \mathrm{C}$. These properties have so far prevented its use as a structural material for hot section loadbearing components.

In particular, the drive towards advanced high thrust-to-weight ratio propulsion systems requires high strength and low-density structural materials capable of extended operation at temperatures up to $1600^{\circ} \mathrm{C}[3,4]$, which is clearly above the operation temperature of Ni-based superalloys used nowadays for turbine airfoils in aeroplane engines (operation temperature below $1000^{\circ} \mathrm{C}$ ). The creep properties of $\mathrm{MoSi}_{2}$ may be improved by using it together with a second material, e.g. $\mathrm{SiC}$ or $\mathrm{WSi}_{2}$, in a composite or an alloy. A new class of silicide matrix composites designed around $\mathrm{MoSi}_{2}$ appears to provide an alternative to Si-based ceramics such as $\mathrm{SiC}$ and $\mathrm{Si}_{3} \mathrm{~N}_{4}$. Projected applications of $\mathrm{MoSi}_{2}$-based materials include hot section components in aerospace gas turbines, glow plugs of diesel engines and industrial gas burners. For a review of $\mathrm{MoSi}_{2}$-based materials, their properties and their creep behaviour see the reviews of Vasusdevan and Petrovic [3], Yao et al. [4] and the original papers of Sadananda et al. [5, 6]. For creep of other binary intermetallics the reader may consult Chapter 16 in Westbrook and Fleischer [7].

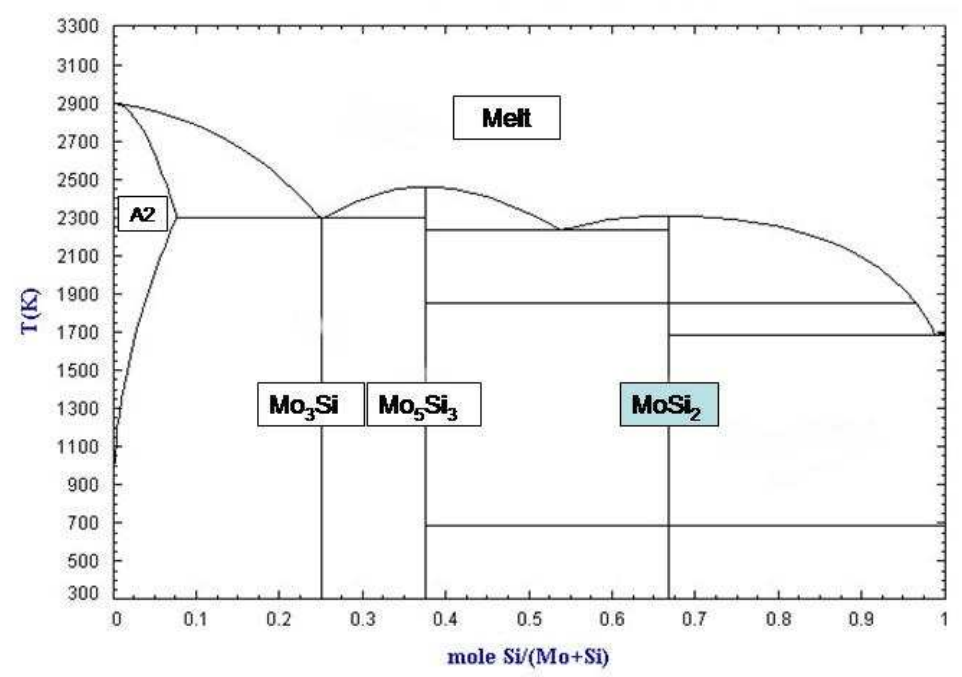

Figure 1 Phase diagram of the binary molybdenum-silicon system. 
The phase diagram of the binary Mo-Si system shows that $\mathrm{MoSi}_{2}$ is a line compound with very weak deviations from stoichiometry. Formerly, it was believed that $\mathrm{MoSi}_{2}$ crystallises in a tetragonal structure below $1900^{\circ} \mathrm{C}$ ( $\alpha$-phase) and that a hexagonal high-temperature phase ( $\beta$-phase) exists between $1900^{\circ} \mathrm{C}$ and the melting temperature. However, more recent investigations showed that the tetragonal structure is thermodynamically stable up to the melting temperature, whereas the hexagonal $\beta$-phase is stabilised by impurities [8].

The crystal structure of $\mathrm{MoSi}_{2}$ is tetragonal of $\mathrm{C} 11_{\mathrm{b}}$ type, space group 14/mmm (see Fig.2). The lattice parameters are $a=0.3205 \mathrm{~nm}$ and $c=0.7845 \mathrm{~nm}$ with $c / a=2.45$. Molybdenum atoms are located at the corners and in the centre of the tetragonal cell. Silicon atoms are located at $(0,0$, $1 / 3),(0,0,2 / 3),(1 / 2,1 / 2,1 / 6)$ and $(1 / 2,1 / 2,5 / 6)$ positions. The tetragonal cell can be considered as consisting of three squashed cubic pseudo-cells stacked in c-direction, each pseudo-cell containing an atom in its body centre. The structure may also be viewed as atomic square-lattice layers stacked in the tetragonal direction and shifted by $(1 / 2,1 / 2,1 / 6)$. One layer of Mo atoms is followed by two layers of Si atoms. Each Mo atom is surrounded by a 'cage' of ten Si atoms. In contrast, each $\mathrm{Si}$ atom has five Si atoms and five Mo atoms as neighbors.

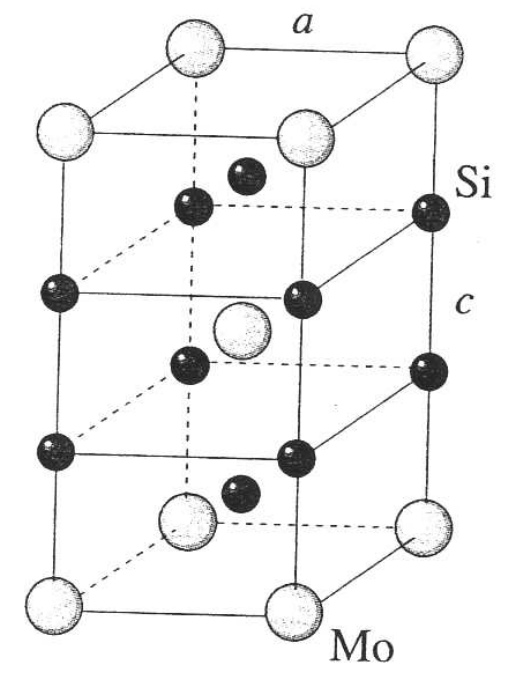

Figure 2 The tetragonal unit cell of $\mathrm{C} 11_{\mathrm{b}}$ structured molybdenum disilicide. $a$ and $c$ denote the lattice parameters of the tetragonal cell.

Among the physical properties for future research and development directions of $\mathrm{MoSi}_{2}-$ based composites diffusion is a crucial one. Diffusion is ubiquitous at high temperatures and selfdiffusion is the most basic diffusion process. It is relevant for an understanding of creep rates and for the formation of corrosion-protecting $\mathrm{SiO}_{2}$ layers. Creep is a phenomenon primarily of engineering interest. Minimizing the creep rate is of paramount importance.

Until recently, no reliable diffusion data for $\mathrm{MoSi}_{2}$ were available. Fortunately, this situation changed due to the work from the Münster laboratory by Salamon and coworkers [9-13], when the self-diffusion of both components $\mathrm{Si}$ and Mo, and also diffusion of the foreign element Ge, which is tetravalent like $\mathrm{Si}$, was studied. Further progress came from positron annihilation studies on $\mathrm{MoSi}_{2}$ by Zhang et al. [14], which provided insight into the type of defects present in thermal equilibrium. In the following two sections we summarize the information that was provided by the diffusion and by positron annihilation studies.

The evidence about diffusion and thermal defects strongly suggests that diffusion of Si and Mo occurs by vacancies in the respective sublattices. It is long known that vacancy-mediated diffusion is associated with correlation effects. Correlation effects of Si self-diffusion in $\mathrm{MoSi}_{2}$ have been calculated recently using a combination of a matrix method and Monte Carlo simulations by Divinski et al. [16]. Belova et al. [17] treated both, Si and Mo diffusion, using direct Monte Carlo 
simulations. We mention the major results of these calculations in the section on diffusion in $\mathrm{MoSi}_{2}$. Finally, we remind the reader first about some basic facts of high-temperature creep. Hightemperature creep is closely related to self-diffusion. Therefore we discuss creep deformation data for $\mathrm{MoSi}_{2}$ available from the work of Sadananda et al. [5, 6] in the light of the now available information about defects and diffusion.

\section{Diffusion in Molybdenum Disilicide}

Review of Diffusion Studies. A comprehensive diffusion study for a tetragonal material requires measurements of the diffusivity in both principal directions of the crystal structure. The diffusion coefficient in an arbitrary direction is then given by

$$
D=D \perp \sin ^{2}(\theta)+D \| \cos ^{2}(\theta)
$$

where $\theta$ is the angle between diffusion direction and the tetragonal axis. $D \|$ and $D \perp$ denote the principal diffusivities parallel and perpendicular to the tetragonal axis.

Self-diffusion of Mo and Si and diffusion of the foreign atom Ge in oriented single crystals of $\mathrm{MoSi}_{2}$ have been studied by the Münster group and the principal diffusivities in both directions have been deduced [9-13]. The radiotracer technique, which is considered to be the most reliable method for self- and impurity diffusion studies, was used in these experiments. Self-diffusion was studied with the radioisotopes ${ }^{99} \mathrm{Mo}$ and ${ }^{31} \mathrm{Si}$, which has a half-life of 2.6 hours only. The study of Si diffusion and in particular its temperature range is limited by the short half-life of this isotope. The radioisotope ${ }^{71} \mathrm{Ge}$ was used because tetravalent $\mathrm{Ge}$ is suitable to mimic Si self-diffusion.

In the Arrhenius diagram of Fig. 3 the diffusivities of all three isotopes in both principal directions are displayed. Silicon and Ge diffusion coefficients are many orders of magnitude higher than the Mo diffusivities. For example, near $1500 \mathrm{~K}$ the difference between the diffusivities is about six orders of magnitude. This huge difference between the diffusivities of the components of a binary intermetallic compound is remarkable. To the authors' knowledge no other intermetallic reveals such a large difference between the diffusivities of its constituents. Such differences between component diffusivities are typical for ceramic materials and metal oxides, in which the diffusion of the components is often restricted to the sublattices of the components. A well-studied example is the oxygen ion conductor yttrium doped zirconia $\left(\mathrm{ZrO}_{2} 9.5 \mathrm{~mol} \% \mathrm{Y}_{2} \mathrm{O}_{3}\right)$. In single crystals, oxygen diffusion is more than eight orders of magnitude faster than $\mathrm{Zr}$ diffusion. Both diffusivities are enhanced by orders of magnitude in the interfaces of the nanocrystalline material (see, e.g., Schaefer [15]):

For all three diffusants in $\mathrm{MoSi}_{2}$ the diffusivities perpendicular to the tetragonal axis are higher than parallel to it. The magnitude of the diffusion anisotropy is also remarkable. Diffusion of Ge and Si occur at similar rates, confirming the idea that Ge is a suitable atom to mimic Si selfdiffusion. The slightly faster Ge diffusion is presumably due to an attractive interaction between Ge atoms and the diffusion-mediating defect, which is for Si and Ge (see below) the vacancy in the $\mathrm{Si}$ sublattice. 


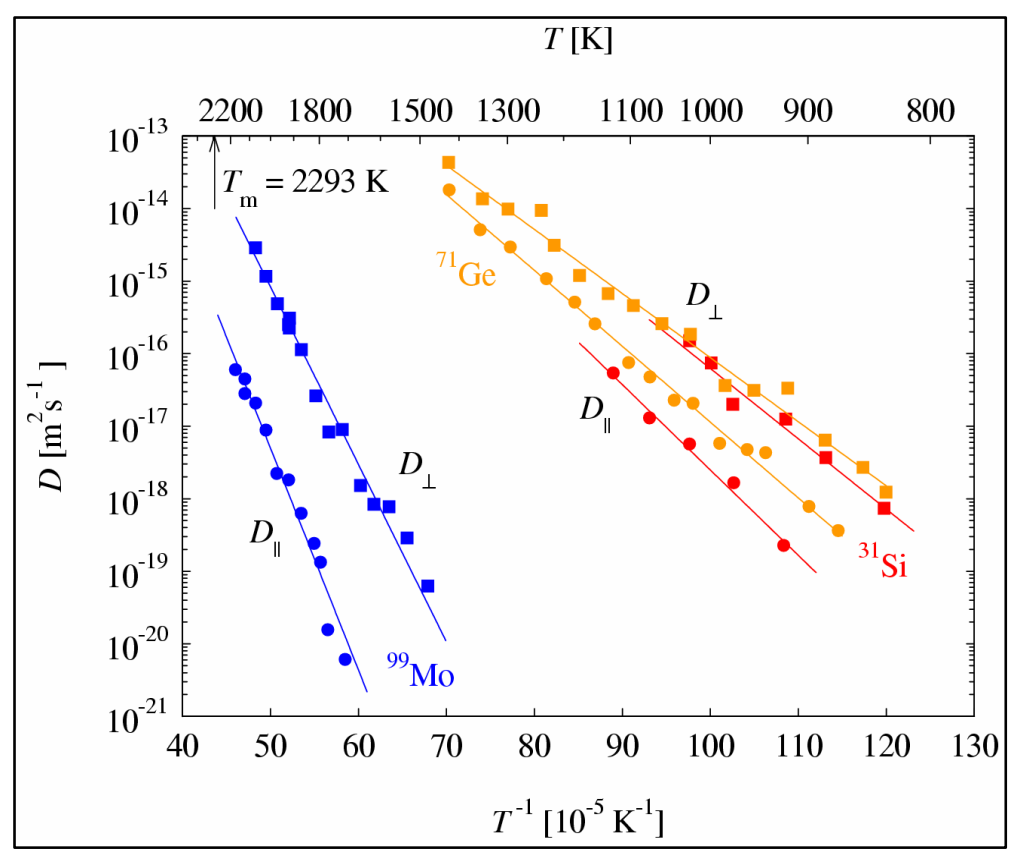

Figure 3 Tracer diffusion of ${ }^{71} \mathrm{Ge},{ }^{31} \mathrm{Si}$, and ${ }^{99} \mathrm{Mo}$ in $\mathrm{MoSi}_{2}$ perpendicular and parallel to the tetragonal axis according to Salamon and Mehrer [11]. Indicated by an arrow is the temperature range of positron anni-hilation studies of Zhang et al. [14] discussed in the text.

Theoretical Expressions for the Principal Self-diffusivities. In what follows, we describe diffusion on an atomic level in the crystal lattice of $\mathrm{MoSi}_{2}$. This description is based on the assumption that diffusion of $\mathrm{Si}$ or Mo atoms are restricted to the $\mathrm{Si}$ or Mo sublattices and that diffusion is mediated in both sublattices by sublattice vacancies. Strong arguments that support this assumption are given below in the section about vacancies studied by positron annihilation.

For a tetragonal crystal of a binary compound like $\mathrm{MoSi}_{2}$, self-diffusion of each component exhibits two principal diffusivities. The (shortest) atomic jumps in the Si and Mo sublattices, which enable diffusion of $\mathrm{Si}$ and Mo atoms on their respective sublattices by exchange with sublattice vacancies are indicated in Fig. 4. Type 1 and type 2 jumps promote Si diffusion in the Si sublattice; type 1 jumps contribute to diffusion in both principal directions, whereas type 2 jumps displace $\mathrm{Si}$ atoms parallel to the tetragonal axis only. Type 3 and type 4 jumps promote Mo diffusion in the Mo sublattice; type 3 jumps contribute to Mo diffusion perpendicular to the tetragonal axis only, whereas type 4 jumps contribute to Mo diffusion in both directions. We use the symbols defined below:

$C_{V}(\mathrm{Si}), C_{V}(\mathrm{Mo}): \quad$ vacancy concentrations (in site fractions) on the Si sublattice or the Mo sublattice

$\omega_{1}$ and $\omega_{2}: \quad$ jump rates of Si atoms for type 1 or type 2 exchange jumps with a vacancy on a neighbour site of the Si sublattice (see Fig. 4)

$\omega_{3}$ and $\omega_{4}: \quad$ jump rates of Mo atoms for type 3 or type 4 exchange jumps with a vacancy on a neighbor site of the Mo sublattice (see Fig. 4)

$D \|(S i), D \Perp(S i): \quad$ diffusivities parallel and perpendicular to the tetragonal axis in the $\mathrm{Si}$ sublattice

$D \|(M o), D \unrhd(M o)$ : diffusivities parallel and perpendicular to the tetragonal axis in the Mo sublattice 
$f \|(S i), f \unrhd(S i): \quad$ correlation factors for diffusion of Si parallel and perpendicular to the tetragonal axis

$f \|(M o), f \unrhd(M o): \quad$ correlation factors for diffusion of Mo parallel and perpendicular to the tetragonal axis

Using these quantities, the tracer diffusivities of Si diffusion in the Si sublattice can be written as follows:

$$
D_{\|}(S i)=(1 / 2)(c / 3)^{2}\left(\omega_{1}+\omega_{2}\right) f_{\|}(S i) C_{V}(S i) \text { and } D \Perp(S i)=(1 / 2) a^{2} \omega_{1} f_{\Perp}(S i) C_{V}(S i) .
$$

For the tracer diffusivities of Mo diffusion in the Mo sublattice we obtain:

$$
D_{\|}(M o)=c^{2} \omega_{4} f \|(M o) C_{V}(M o) \text { and } D \perp(M o)=a^{2}\left(\omega_{3}+\omega_{4}\right) f \Perp(M o) C_{V}(M o) .
$$

We may introduce the anisotropy ratio for Si diffusion, e.g., in the following way:

$$
D_{\Perp}(S i) / D_{\|}(S i)=(1 / 9)(a / c)^{2}\left[\omega_{2} /\left(\omega_{1}+\omega_{2}\right)\right]\left[f_{\Perp}(S i) / f \|(S i)\right) .
$$

For the anisotropy ratio of Mo diffusion we find:

$$
D \perp(M o) / D_{\|}(M o)=(a / c)^{2}\left[\left(\omega_{3}+\omega_{4}\right) / \omega_{4}\right] f_{\Perp}(M o) / f_{\|}(M o) .
$$

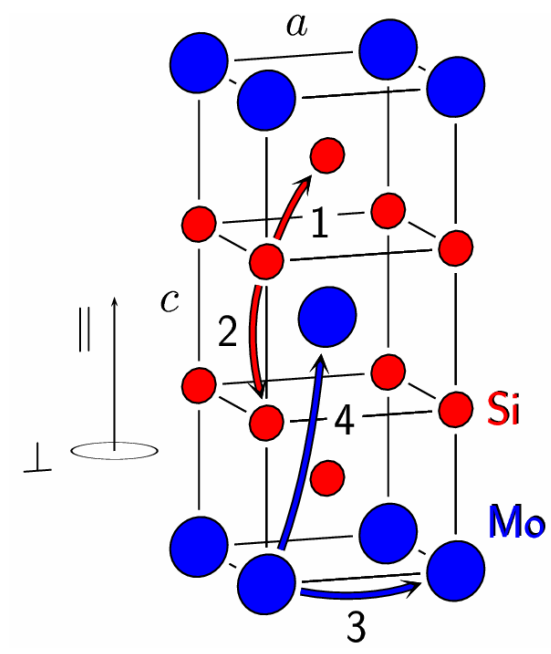

Figure 4 The atomic jumps in the Si and Mo sublattices of molybdenum disilicide are indicated in the tetragonal unit cell. These jumps enable diffusion of Si and Mo atoms on their respective sublattices. Type 1 and type 2 jumps promote Si diffusion in the Si sublattice; type 3 and type 4 jumps promote Mo diffusion in the Mo sublattice.

Correlation Factors. It is known since the pioneering work of Bardeen and Herring [18] that atomic diffusion via a vacancy mechanism is correlated. Analytical methods for the calculation of correlation factors have been described in the literature [19-21]. Monte Carlo methods are also well suitable to calculate the correlation factors of self-diffusion (see, e.g., Murch [22]). Correlation factors of vacancy-mediated self-diffusion in the $\mathrm{Si}$ sublattice are available from the work of Divinski et al. [16], in which the authors used a combination of analytical and Monte Carlo approaches. Correlation factors and anisotropy ratios for tracer self-diffusion in both sublattices have been deduced by direct Monte Carlo techniques very recently by Belova et al. [17]. For the correlation effects in the Si sublattice, which were treated in both papers, the results are in good agreement. Eqs. (4) and (5) yield the anisotropy ratios of self-diffusion for both sublattices. 
Considering the jump rates illustrated in Fig.4, the correlation factors and the anisotropy ratios in Eqs.(2) to (5) are functions of the ratios of the two jump rates in the respective sublattice:

The correlation factors for the Si sublattice are displayed in Fig. 5. This figure shows that the correlation factor for Si diffusion parallel to the tetragonal axis approaches zero for large and small values of the ratio $\omega_{2} / \omega_{1}$. When the jump rate $\omega_{2}$ is much smaller or much higher than $\omega_{1}$ no long-range diffusion parallel to the tetragonal axis can occur (see Fig. 4) and hence $\boldsymbol{f}_{\|}(\mathrm{Si})$ approaches zero. Only if the ratio of the two jump rates is not too much different from unity, the tracer can be transported parallel to the tetragonal axis over distances which exceed the jump length. The correlation factor $\boldsymbol{f} \doteq(\mathbf{S i})$ approaches constant but different values for very large or very small values of the ratio of the two jump rates. In the interval $10^{-2}<\omega_{2} / \omega_{1}<10^{2}$ the two correlation factors vary significantly with the ratio of the two jump rates. If the ratio of the jump rates lies in this interval, the correlation factors contribute to the temperature dependences of the Si diffusivities parallel and perpendicular to the axis and hence to the pertaining activation enthalpies of diffusion.

A comparison between the experimental data for Si diffusion and the numerical results has been reported in $[16,17]$. It is interesting to note, that the correlation factor of Si diffusion parallel to the axis is not a constant but is temperature dependent according to the following expression

$$
\left.f_{\|} / \mathrm{Si}\right) \approx 2.23 \omega_{2} / \omega_{1}=3.17 \exp \left(-39 \mathrm{~kJ} \mathrm{~mol}^{-1} / k_{B} T\right)=3.17 \exp \left(-0.40 \mathrm{eV} / k_{B} T\right) \text {. }
$$

This temperature dependence contributes about $17 \%$ to the activation enthalpy of Si diffusion parallel to the tetragonal axis.

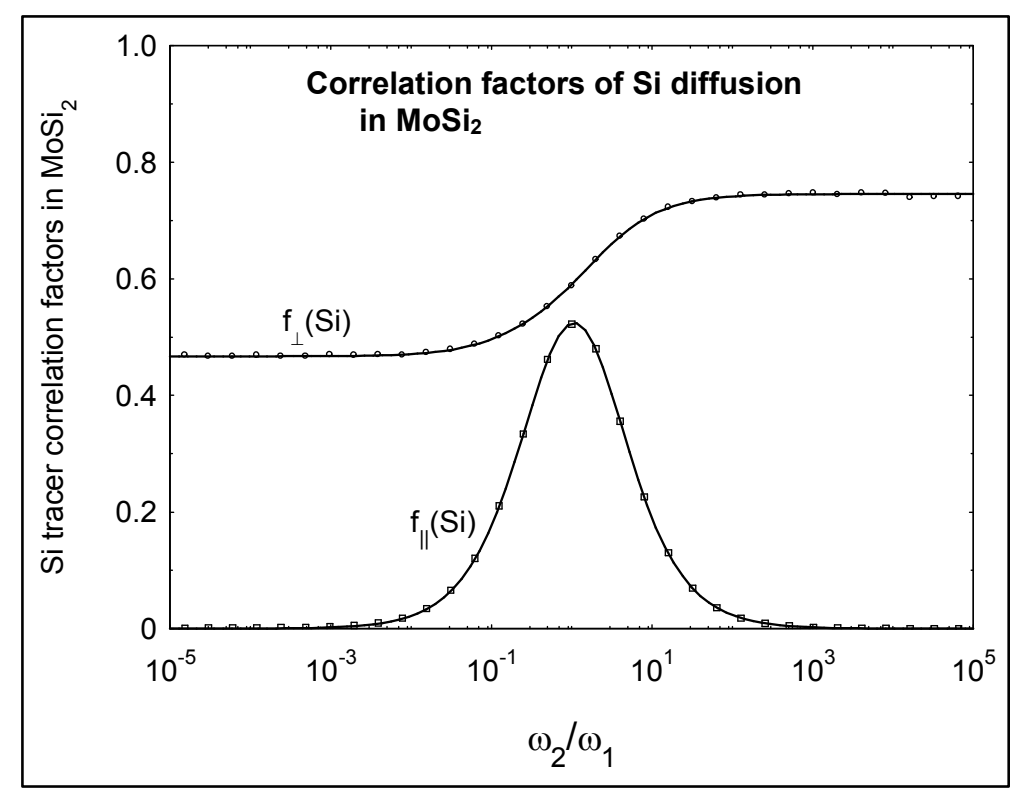

Figure 5 Correlation factors for Si diffusion parallel and perpendicular to the tetragonal axis of molybdenum disilicide as functions of the ratio $\omega_{2} / \omega_{1}$. Full lines represent the results of Divinski et al. [16]. The dots represent the results of Belova et al. [17]. The two calculations are in good agreement.

The correlation factors for the Mo sublattice are displayed in Fig. 6. This figure shows that for ratios $\omega_{4} / \omega_{3}$ very large or very small both correlation factors approach constant values. If the ratio becomes very large, i.e. when $\omega_{4}$ jumps dominate, both tracer correlation factors approach the bcc value of 0.727 . When $\omega_{3}$ jumps dominate, the correlation factor perpendicular to the tetragonal axis approaches the limit 0.467 , which corresponds to the value of the planar square lattice. The correlation factor parallel to the tetragonal axis tends to unity. In this limit, the vacancy has enough freedom to approach the tracer from a random direction along the tetragonal axis. If the ratio of the 
jump rates lies in the interval $10^{-2}<\omega_{4} / \omega_{3}<10^{2}$, the correlation factors contribute to the temperature dependences of the Mo diffusivities parallel and perpendicular to the axis and hence to the pertaining activation enthalpies of diffusion.

According to the experimental results of Fig.3, the anisotropy ratio $D \perp(M o) / D \|(M o)$ is about 100 or larger in the temperature range of the experiments. From Eq. (5) then follows that $\omega_{3}$ jumps must be about a factor of 100 more frequent than $\omega_{4}$ jumps. In this range, Fig. 6 shows that the correlation factors are practically constant and hence do not contribute to the temperature dependence of the Mo diffusivities.

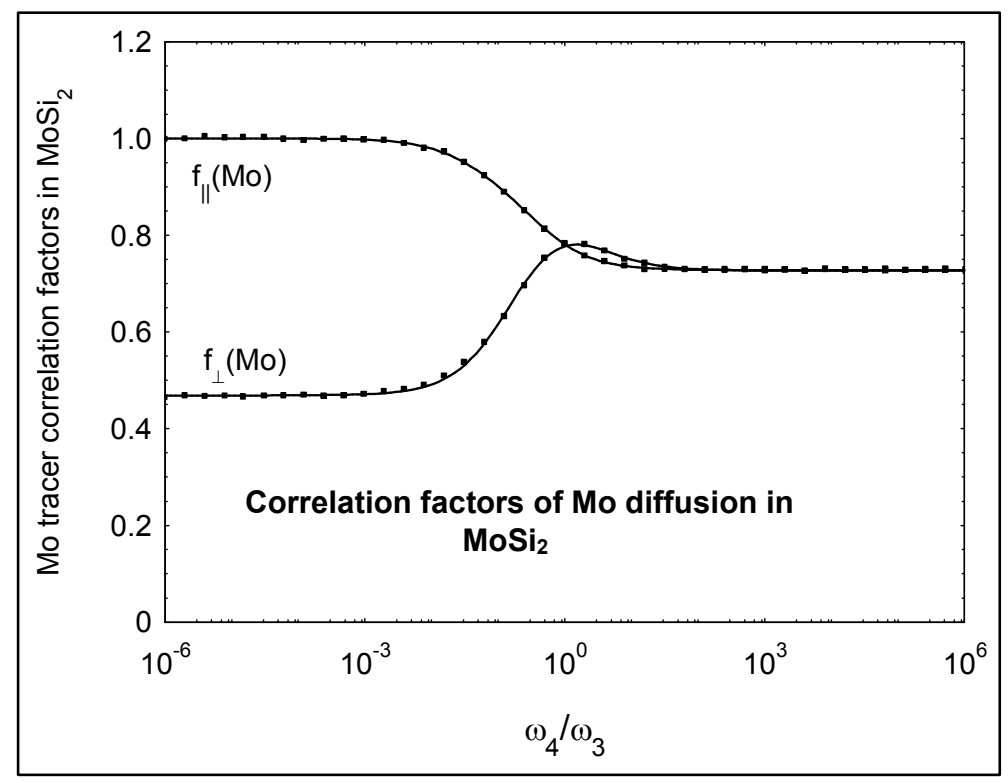

Figure 6 Correlation factors for Mo diffusion parallel and perpendicular to the tetragonal axis of molybdenum disilicide as functions of the ratio $\omega_{4} / \omega_{3}$ from Belova et al. [17].

\section{Vacancies in molybdenum disilicide}

For the high-temperature properties of materials vacancies that are present in thermal equilibrium are important and can be studied by positron annihilation techniques (see, e.g., Schaefer et al. [23, 24]). In particular they are the vehicles of diffusion. Vacancies located on the Si and the Mo sublattice are schematically indicated in Fig. 7. This figure shows that Si and Mo vacancies have different surroundings. A vacancy on the Mo sublattice has only Si atoms as nearest neighbors whereas a vacancy on the Si sublattice has $\mathrm{Si}$ and Mo neighbors. In a binary compound like $\mathrm{MoSi}_{2}$ vacancies may be formed on each sublattice with different formation enthalpies and hence appear with significant site fractions in different temperature ranges.

For the investigation of thermal vacancies in materials the annihilation of positrons with electrons of the solid and the detection of the annihilation radiation is a sensitive technique. In such studies, positrons from proton-rich radioisotopes with simultaneously emitted decay $\gamma$-quanta are used as positron sources.

In positron lifetime experiments, the time delay between the decay quantum and the positron-electron annihilation $\gamma$-quanta is used to deduce the positron lifetime. Usually the lifetime increases when the positron gets trapped in a vacancy because the valence electron density in a vacancy is lower than in interstitial sites of the perfect lattice. Therefore, when in a solid thermal vacancies are formed the mean positron lifetime increases in an S-shaped manner with increasing temperature. From an analysis of such data the formation enthalpy of vacancies can be deduced. 
For binary compounds it is difficult to tell from lifetime studies on which sublattice vacancies predominantely form. Fortunately, the annihilation quanta of positron and electron of the solid carry also information on the momentum of the electron. Coincident Doppler broadening spectra can be used for a measurement of the electron momentum distribution up to the relatively high momenta of the core electrons. In this way, the atoms surrounding a vacancy can be analysed and thereby the sublattice on which a vacancy is formed in an ordered intrmetallic compound may be identified. A study of thermal vacancies in $\mathrm{MoSi}_{2}$ and particularly the sublattice of the compound on which vacancies are formed is of particular interest.

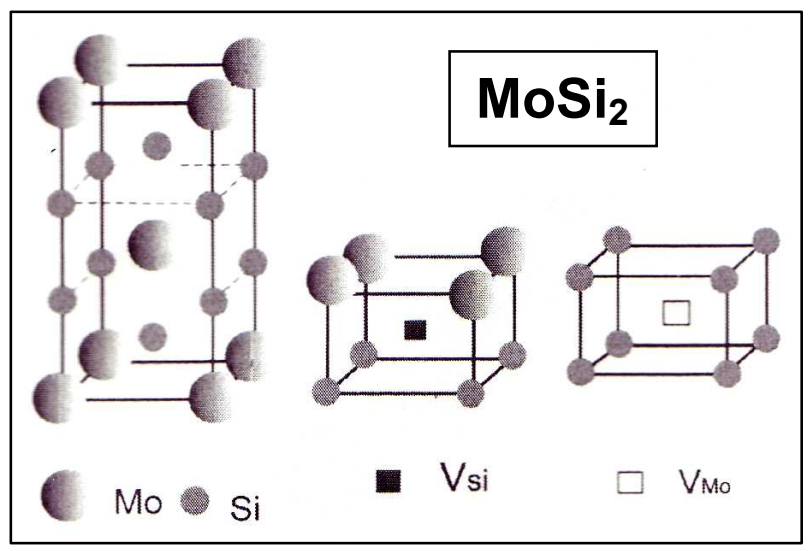

Figure 7 Perfect lattice of molybdenum disilicide (left), vacancy on the Si sublattice (middle), and vacancy on the Mo sublattice (right) according to Zhang et al. [14].

Molybdenum disilicide has been studied by both positron annihilation techniques by several authors. Fig. 8 shows the mean positron lifetime as a function of temperature from the work of Zhang et al. [14]. Below $1050 \mathrm{~K}$ the positron life time shows a weak temperature dependence. This part is attributed to the free positron lifetime (positron annihilation from an interstitial site of the crystal lattice), which increases slightly with temperature to due the thermal expansion of the lattice. A reversible S-shaped increase of the mean positron lifetime is observed between 1050 and $1343 \mathrm{~K}$, the highest temperature studied. This has been attributed to positron trapping at thermal vacancies in one type of vacancies with a positron lifetime of $(165 \pm 5)$ ps.

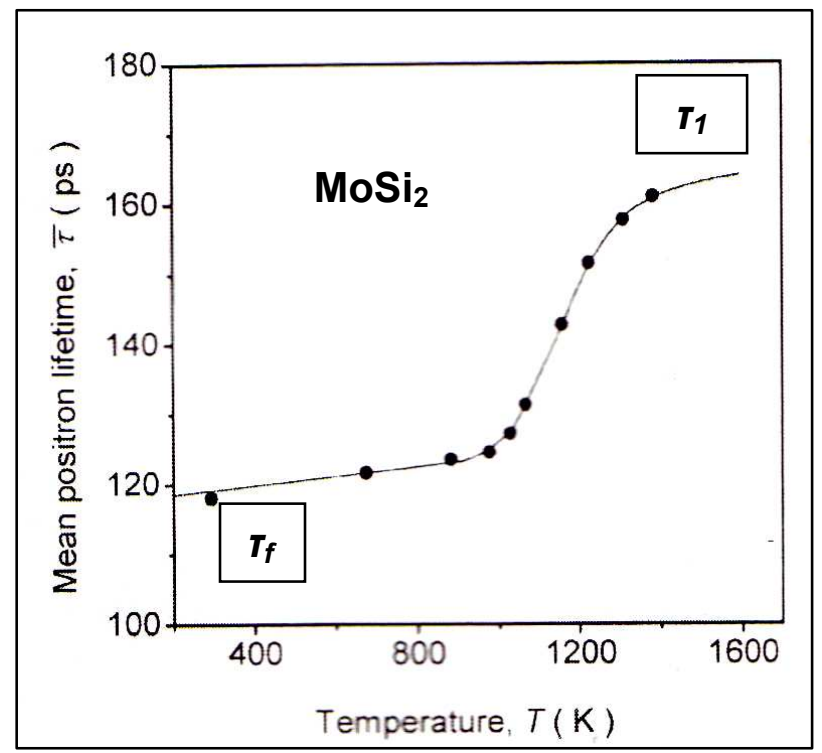

Figure 8 Mean positron lifetime in molybdenum disilicide as a function of temperature according to Zhang et al. [14]. The solid line is a fit of the two-state positron trapping model (see Eq.(7)) to the data. 
Lifetime spectroscopy indicated that in well-annealed $\mathrm{MoSi}_{2}$ structural vacancies do not exist. The major merits of lifetime spectroscopy, however, concern the detection of thermal vacancies. For the two-state positron trapping model the variation of the mean positron lifetime $\bar{\tau}$ is given by

$$
\overline{\tau(T)}=\tau_{f} \frac{1+\sigma_{c} \exp \left(S_{V}^{F} / k_{B}\right) \exp \left(-H_{V}^{F} / k_{B} T\right) \tau_{1}}{1+\sigma_{c} \exp \left(S_{V}^{F} / k_{B}\right) \exp \left(-H_{V}^{F} / k_{B} T\right) \tau_{f}},
$$

where $\tau_{f}$ is the positron lifetime in the defect-free crystal, with a weak temperature dependence given by $\tau_{f}=\tau_{f 0}(1+\alpha T)$, where $\alpha$ is a thermal expansion coefficient similar to that of $\mathrm{MoSi}_{2}$ and $\tau_{f 0}=117 p s$ the positron lifetime at ambient temperature. $\sigma_{c}$ is the specific positron trapping rate, $H_{V}^{F}$ and $S_{V}^{F}$ denote the formation enthalpy and entropy of a vacancy, and $\tau_{1}$ is the lifetime of the positron trapped in a vacancy. The formation of thermal vacancies obtained from a fit to the data yielded a formation enthalpy of about $(1.6 \pm 0.1) \mathrm{eV}$ or $154 \mathrm{kJmol}^{-1}$. The annealing of radiationinduced point defects was observed in poasitron annihilation studies by Matsuda et al. [33]. The authors found no indication for structural vacancies

For identifying the sublattice on which thermal vacancies are formed Zhang et al. [14] also performed coincident measurements of the Doppler broadening of the $\gamma$-quanta emitted by positron annihilation at $1343 \mathrm{~K}$. This temperature was chosen because then, according to Fig. 8, the great majority of positrons are trapped in vacancies and hence annihilate from this state. It turned out that the Doppler broadening data can be modelled much better for vacancies on the Si sublattice (see Fig. 9). This demonstrates that thermal vacancies up to the highest temperatures studied in the positron experiments are predominantly formed on the Si sublattice.

A contribution of thermal vacancies on the Mo sublattice could not be discerned in the positron studies in the temperature range investigated. This suggests that the concentration of thermal vacancies on the Mo sublattice is much smaller than on the Si sublattice and the formation enthalpy of vacancies on the Mo sublattice is higher than that of vacancies on the Si sublattice. The independent formation of vacancies in the two sublattices would imply that the composition of $\mathrm{MoSi}_{2}$ may slightly deviate from stoichiometry. Since thermal vacancy fractions are small (e.g. in metals near the melting temperature less than $10^{-3}$ ), such a deviation would be hardly visible in the phase diagram of the Mo-Si system. 


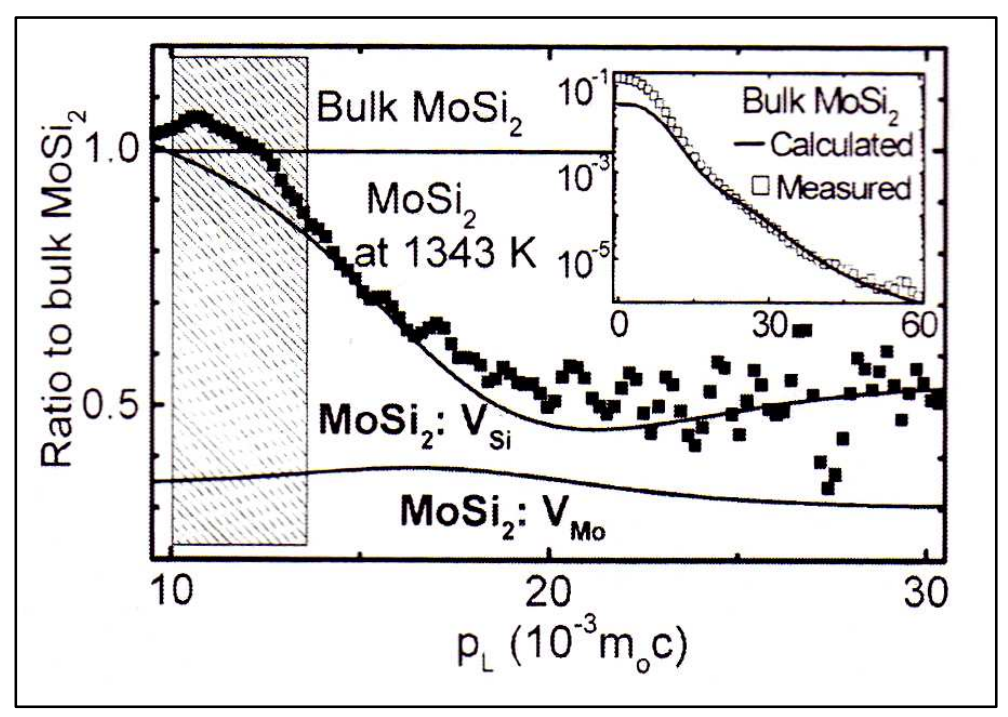

Figure 9 Coincident Doppler-broadened spectrum measured on molybdenum disilicide at $1343 \mathrm{~K}$ according to Zhang et al. [14]. Full squares correspond to positron saturation trapping at vacancies.

Full lines are calculated for vacancies on the Mo sublattice and on the Si sublattice. Agreement between full squares and the calculated line for vacancies on the Si sublattice is evident.

The diffusion data of Fig. 3 with the huge asymmetry between Mo and Si or Ge diffusion and in particular the drastic difference between self-diffusion of the two components of $\mathrm{MoSi}_{2}$ suggest that Mo diffusion is restricted to the Mo sublattice whereas $\mathrm{Si}$ and Ge diffuse on the $\mathrm{Si}$ sublattice of the $\mathrm{MoSi}_{2}$ structure. In addition, the positron experiments indicate that diffusion in the Si sublattice is mediated by thermal vacancies. In what follows, we assume that Mo diffusion occurs via ex-change with thermal vacancies on the Mo sublattice as well. This is not in contradiction with the positron experiments, from which one can only conclude that the concentration of thermal vacancies on the Mo sublattice is much smaller than concentration of $\mathrm{Si}$ sublattice vacancies. This is supported by the low Mo diffusivities of Fig. 5, which are in accord with low concentrations of thermal vacancies in the Mo sublattice. The reader may note that the Mo diffusion data of Fig. 3 have been measured at temperatures above the temperature range of the positron annihilation experiments.

\section{High-temperature Creep}

Basics of High-temperature Creep. Deformation of materials at high temperatures, above about half the melting temperature, brings diffusion processes into play. At elevated temperatures materials under loads, which would have caused no deformation at room temperature, start to creep. Creep is a slow, continuous deformation with time. In what follows we first remind the reader to some basics of creep and then consider two phenomena, the analysis of which involves diffusion processes: dislocation creep and diffusional creep. This introduction partly takes advantage of textbooks of Ashby and Jones [25], Philibert [26], Shewmon [27], and Schaumburg [28].

For a solid undergoing creep the strain depends not only on stress but also on temperature and time. If a constant stress is applied a material deforms over a period of time irrespective the stress is greater than or less than the yield strength measured in a conventional tensile test at that temperature. This creep deformation is, in many cases, the limiting factor for the selection of materials for load-bearing components used at high temperatures.

In a creep test, a specimen is loaded in tension or compression at constant temperature and the strain is measured as a function of time. Typical creep curves (see Fig. 10) show three stages that follow the instantaneous elastic strain. During the first stage, called primary creep, the strain- 
rate decreases. In the second stage, called steady-state or secondary creep, the strain-rate is constant and the strain increases steadily with time. In the third or tertiary creep stage the strain-rate increases rapidly until rupture occurs. The initial or primary stage occurs quickly and can be treated in a similar way as elastic deformation. Most of the lifetime of a creeping material is spent in the steady-state range. Therefore, it is this range that dominates the lifetime of a structure.

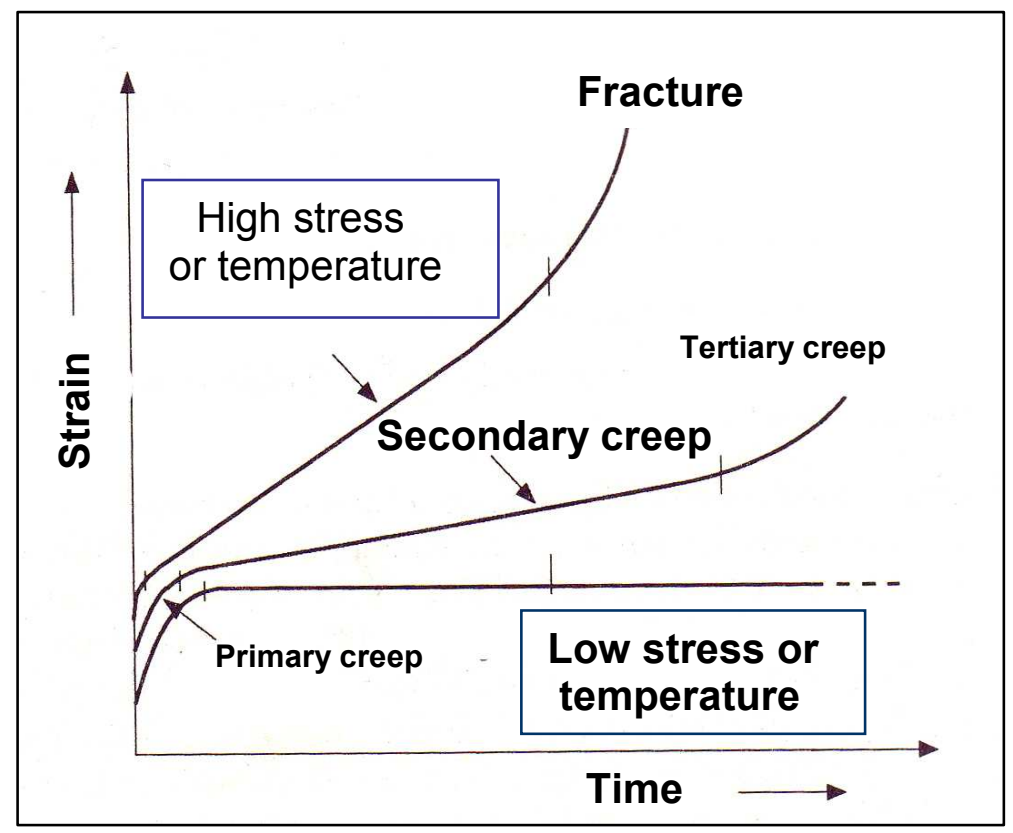

Figure 10 Schematic illustration of creep curves of a solid.

Power-law or Dislocation Creep. Creep is a stress-dependent and thermally activated process. It is accelerated by an increase of applied stress $\sigma$. The steady-state creep rate (index ss) at constant temperature and at not too low stresses is given by

$$
d \varepsilon_{s s} / d t=B \sigma^{n}
$$

Here $n$ is denoted as creep exponent and usually lies between about 3 and 8 . This sort of creep is called 'power-law' creep. The constant $B$ is thermally activated with an activation enthalpy $Q$. Introducing this into Eq. (8) for the creep rate, power-law creep can be written as

$$
d \varepsilon_{s s} / d t=A \exp \left(-Q / k_{B} T\right) \sigma^{n}
$$

As usual $k_{B}$ denotes the Boltzmann constant and $T$ absolute temperature. The values $A, n$, and $Q$ are determined experimentally and vary from material to material.

Power-law creep is attributed to dislocation creep. When a crystalline material deforms plastically, dislocations have to overcome the intrinsic lattice resistance and the effect of obstacles such as solute atoms, precipitates or other dislocations. Diffusion of atoms leads to dislocation climb, which can unlock dislocations from obstacles (see Fig. 11). During climb of edge dislocations, atoms diffuse from/to the bottom of the half-plane of the dislocation. At high enough temperatures, diffusion mainly occurs by lattice diffusion. At lower temperatures, dislocation core diffusion (also denoted as dislocation pipe diffusion) becomes important. The unlocked dislocations glide to the next obstacle and the unlocking takes place again. This climb-glide sequence leads to creep. The role of diffusion is to enable dislocation climb. Lattice and/or dislocation core diffusion explains the thermally activated nature of dislocation creep. There are very few systematic data about dislocation pipe diffusion available in the literature (see, Chapter 11 in [30]). A reasonable guess for the activation energy of dislocation pipe diffusion in metals appears to be 70 to $80 \%$ of the activation energy of lattice diffusion 


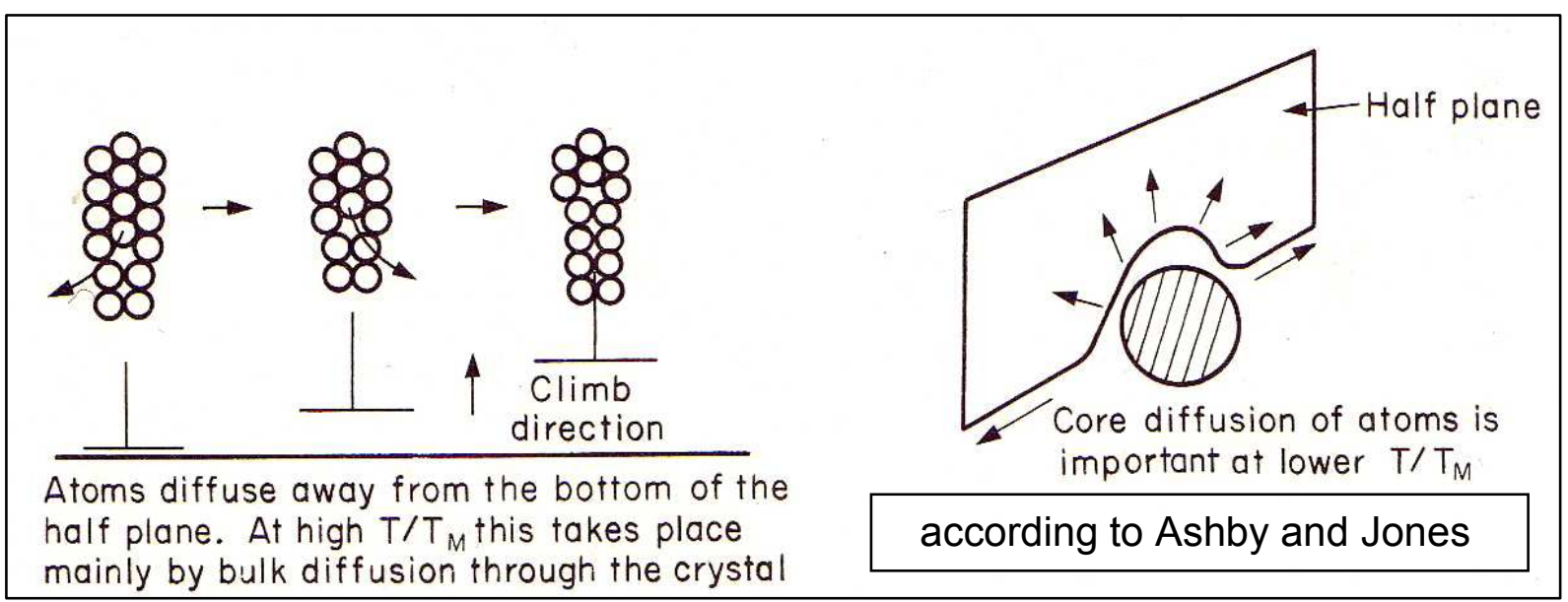

Figure 11 Schematic illustration how lattice (left) and dislocation core (or pipe) diffusion (right) lead to dis-location climb according to Asby and Jones [24].

Diffusional Creep. When the applied stresses are too low to move dislocations, power-law creep stops. In this region creep does, however, not stop but occurs by the diffusion of atoms. This creep mode is called diffusional creep, also denoted as linear viscous or as Newtonian creep. The diffusion can occur by lattice diffusion, in which case it is called Nabarro-Herring creep. If the atomic transport occurs via grain boundaries it is called Coble creep.

Nabarro-Herring creep: For reasons of simplicity, let us consider a polycrystals made of grains of diameter $L$ (see Fig. 12). In a non-isostatic stress field the applied stress can be relieved by elongation of grains. On a boundary normal to the applied stress the chemical potential of atoms is reduced by $-\sigma \Omega$ due to the work that the stress $\sigma$ exerts if an atom with atomic volume $\Omega$ is added to such a boundary, thereby expanding the sample. The stress acts as a mechanical driving force for the diffusion of atoms from one side of a grain to the other. There is no change of the chemical potential at boundaries parallel to the applied stress. Since at high enough temperatures diffusion takes place through the lattice itself the creep rate is then proportional to the lattice diffusion coefficient $D$ :

$$
d \varepsilon_{s s} / d t=B \frac{D}{k_{B} T} \frac{\sigma \Omega}{L^{2}}
$$

This equation is called the Nabarro-Herring equation. $B$ is a numerical factor between 10 and 40 depending on the grain shape and stress rate. The creep rate varies as first power of the stress, and as $1 / L^{2}$, i.e. the reciprocal of the grain-size squared. These features distinguish Nabarro-Herring creep from creep involving grain-boundary diffusion.

One should, however note, that Eq. (10) has been developed for pure metals. Then, the meaning of the diffusivity is that of self-diffusion in the pure metal. For a binary system there are several diffusion coefficients: the tracer self-diffusivities of the two components and two interdiffusion coefficients - either according to Darken-Manning or according to Nernst-Planck. It is not a priori clear, which diffusion coefficient should be used in the Nabarro-Herring equation. For a highly stoichiometric compound we suggest that the Nernst-Planck interdiffusion coefficient is most appropriate. The Nernst-Planck equation for $\mathrm{MoSi}_{2}$ can be written as

$$
D=3<D(\mathrm{Mo})><D(\mathrm{Si})>\Phi /[<D(\mathrm{Mo})>+2<D(\mathrm{Si})>],
$$


where $\langle D(\mathrm{Mo})>$ and $<D(\mathrm{Si})>$ denote suitable averages of the two tracer diffusivities of Mo and $\mathrm{Si}$ of the two principal directions, and $\Phi$ a thermodynamic factor. The Nernst-Planck equation was developed for two mobile species in an ionic crystal, where the condition of charge neutrality requires that the two fluxes are coupled and the net flux is zero. For a line compound the fluxes of the two compounds are coupled as well, otherwise the compound would decompose. We have seen above that in $\mathrm{MoSi}_{2}$ the Mo diffusivities are much slower than the $\mathrm{Si}$ diffusivities. Hence the interdiffusion coefficient in Eq. (11) is mainly given by the slow Mo diffusion.

Coble creep: At lower temperatures, when lattice diffusion is too slow, grain-boundary diffusion can take over. This type of diffusional creep is called the Coble mechanism. If grainboundary diffusion is responsible for the atomic transport, the expression for the strain rate is $B\left(D_{g b} \delta / L k_{B} T\right)\left(\sigma \Omega / L^{2}\right)$, where $D_{g b}$ is the diffusion coefficient along grain boundaries and $\delta$ the grain-boundary width. If this term is added to the Nabarro-Herring equation we obtain the expression for the creep rate when atomic transport occurs by both paths:

$$
d \varepsilon_{s s} / d t=B \frac{D_{g b} \sigma \delta \Omega}{k_{B} T L^{3}}\left[\frac{D L}{3 D_{g b} \delta}+1\right] .
$$

Eq. (12) shows that the dimensionless quantity $\left(D L / D_{g b} \delta\right)$ determines whether lattice or grainboundary diffusion controls the creep rate. The grain-boundary contribution to the creep rate varies as $1 / L^{3}$, which indicates that it becomes more important for smaller grain size. The activation enthalpy of grain-boundary diffusion in metals is typically about 40 to $70 \%$ of the activation enthalpy of lattice diffusion (see, e.g. Chapter 12 in [30] and [31]). If both paths of diffusion contribute to diffusional creep, the apparent activation energy of Coble creep lies between these limits and the apparent activation energy is a weighted average of the activation energies of lattice and grain-boundary diffusion.

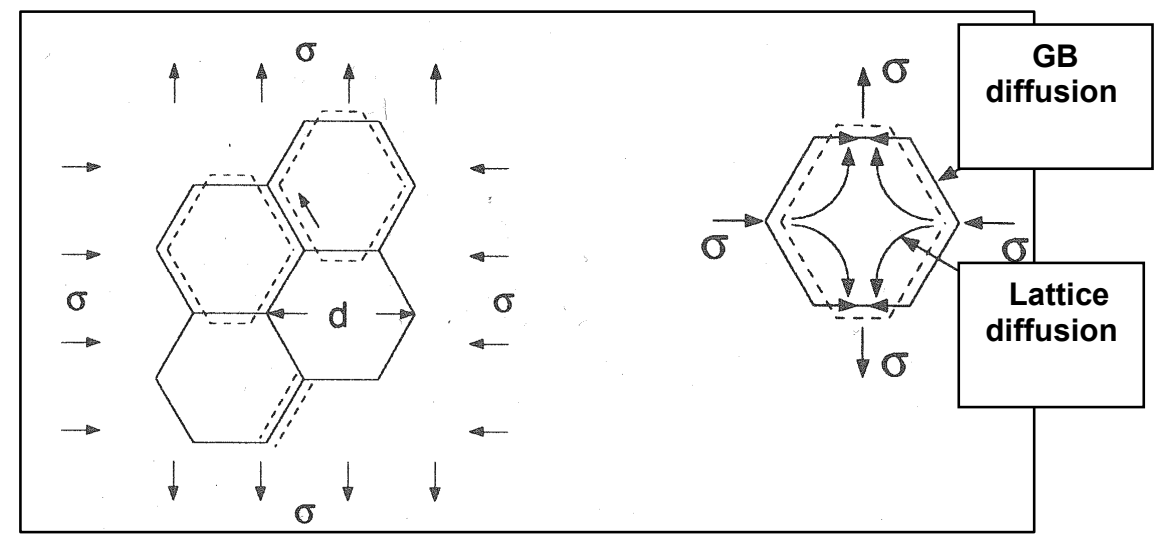

Figure 12 Diffusional or Newtonian creep in a polycrystal (left) and in a single grain (right). Diffusional creep can be mediated either by lattice diffusion or by grain-boundary (GB) diffusion.

Creep and Diffusion of Molybdenum Disilicide. The creep deformation behavior of $\mathrm{MoSi}_{2}$ polycrystals and some of its composites or alloys has been studied in the temperature range between 1100 and $1450^{\circ} \mathrm{C}$ by Sadananda et al. $[5,6]$ and the effects of reinforcement with $\mathrm{SiC}$ and alloying with $\mathrm{WSi}_{2}$ were evaluated. The creep rate of $\mathrm{MoSi}_{2}$ single crystals in soft and hard orientations has been studied by Inui et al. [29] and found to be about two orders of magnitude lower in the hard [001] direction than in the soft [ $\left[\begin{array}{lll}0 & 15 & 1\end{array}\right]$ orientation. The same authors also showed that the creep rate can be lowered by Re doping. 
In what follows we concentrate on the creep behaviour of polycrystalline $\mathrm{MoSi}_{2}$, because these results are most relevant for applications and we perform a comparison of creep and diffusion in $\mathrm{MoSi}_{2}$.

In the first paper by Sadananda et al. [5] the creep rate was studied under compression on polycrystals with fairly large grain size (about 18 to $35 \mu \mathrm{m}$ ) obtained by hotpressing of $\mathrm{MoSi}_{2}$ powders at $1820^{\circ} \mathrm{C}$. The creep rate as a function of the creep strain decreases continuously to a minimum and then remains constant. The first stage corresponds to primary creep. In the second stage, during steady-state or secondary creep, the strain-rate is constant and the strain increases steadily with time. In the rest of the paper we consider steady-state creep.

Fig. 13 shows the creep rate in the secondary stage as a function of applied stress at three different temperatures. The stress exponents are close to 2 and decrease to a value close to 1 at higher temperatures and stresses.

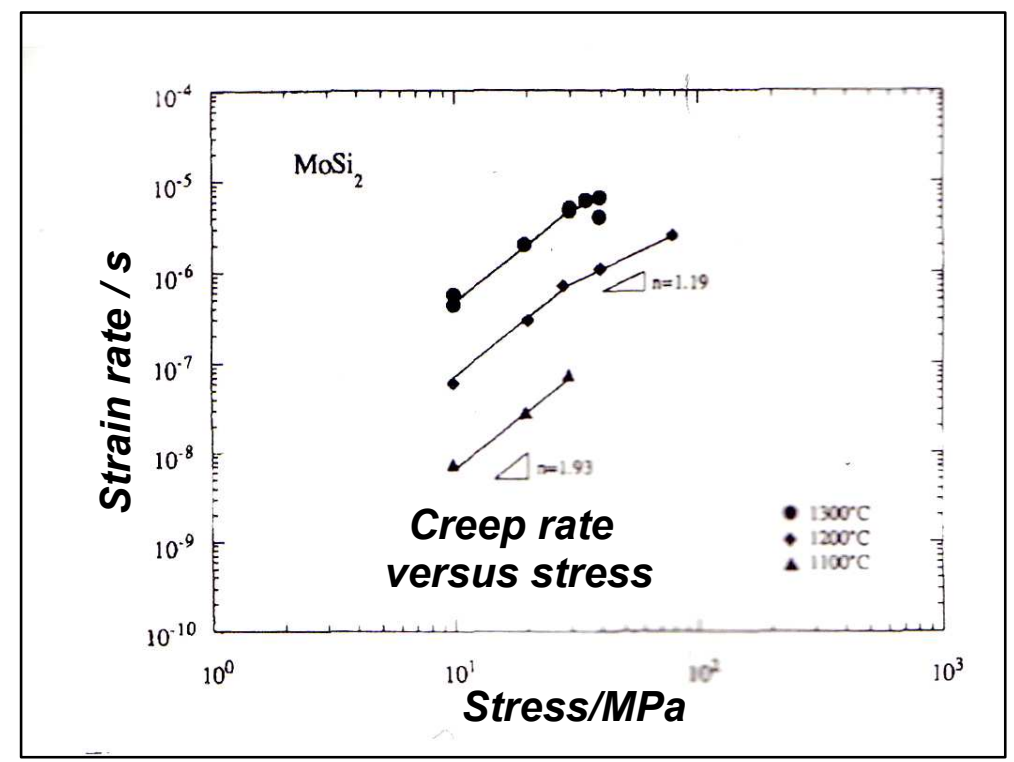

Figure 13 Steady state creep rates of large grain polycrystals of molybdenum disilicide as function of applied stress for various temperatures according to Sadananda et al. [5]. Stress exponents $n=$ 1.93 and $n=1.19$ are indicated.

Apparent activation enthalpies for creep deformation of molybdenum disilicide have been also reported in [5] and are shown in Fig.14. The activation enthalpies are nearly independent of stress in the range investigated. A value of about $433 \mathrm{kJmol}^{-1}$ was obtained from datasets including strain rates at stresses of 10, 20, and $40 \mathrm{MPa}$ only.

Since the associated stress exponents lie between 1 and 2 these observations can be attributed to a transition from diffusional creep to power-law creep, the latter involving dislocation climb. The mechanisms of creep are normally identified on the basis of the strain-rate versus stress exponent and on the basis of the activation enthalpies in comparison with activation enthalpies from diffusion studies. A stress exponent approaching about 3 with increasing temperature is usually attributed to dislocation climb as the rate-controlling process for creep. Stress exponents less than three indicate a transition from diffusional (or viscous or Newtonian) creep to power-law creep, the latter involving dislocation climb with glide. The net creep rate is then determined by a weighted average of the two creep modes. The activation enthalpy for dislocation climb should then correspond either to lattice diffusion or to dislocation pipe diffusion. Diffusional creep can be either mediated at higher temperatures by lattice diffusion or at lower temperatures by grain-boundary diffusion in a polycrystal. 


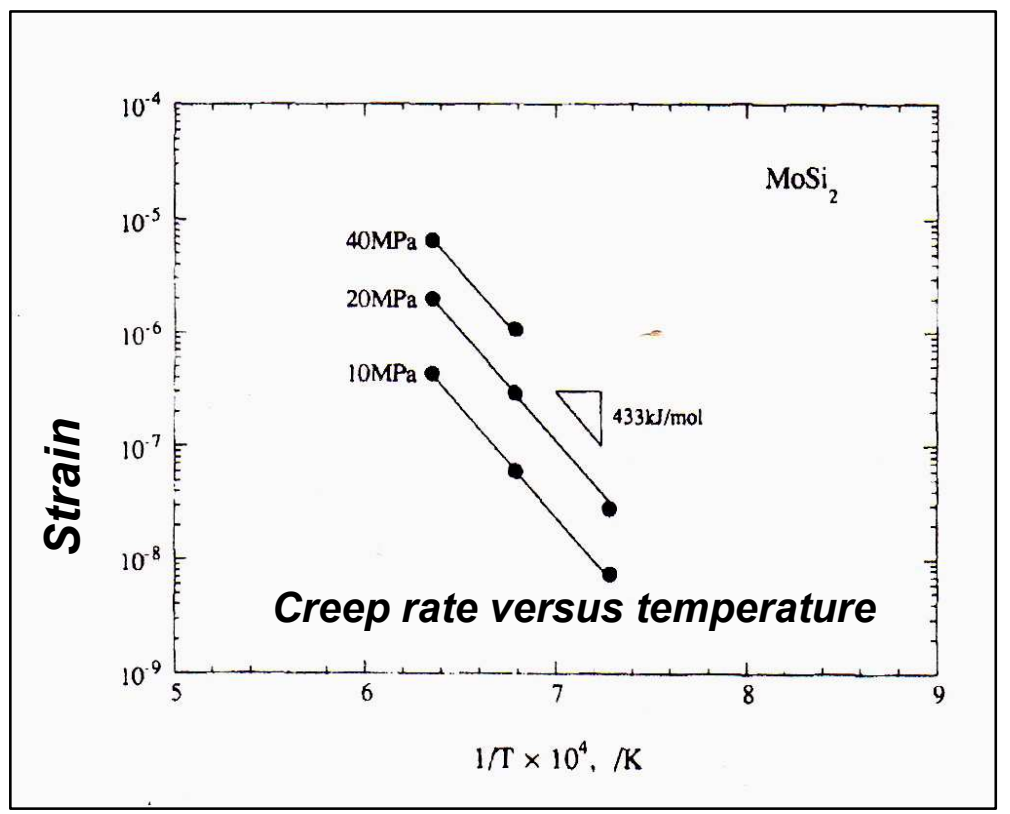

Figure 14 Arrhenius diagram for the determination of activation enthalpies of creep in large grain polycrystals of molybdenum disilicide according to Sadananda et al. [5]. An activation enthalpy of $433 \mathrm{~kJ} / \mathrm{mol}$ is indicated.

A distinction between the two types of diffusional creep would in principle be possible on the basis of the dependence of the Coble equation on the grain size $L$. The creep behaviour has been studied on samples with different grain sizes between 4 and about $30 \mu \mathrm{m}$ by Sadananda et al. [6]. The materials were processed through powder consolidation at various temperatures of the hot press. The grain size effects on creep are significant in these materials, indicating that grain boundaries play an important role in the creep deformation.

Fig. 15 shows the effect of grain size in the Newtonian regime reported in [6]. The creep rates change by three to four orders of magnitude in the range of $L=4$ to $20 \mu \mathrm{m}$ grain size. At a grain size of $20 \mu \mathrm{m}$ Newtonian creep ends at a stress of about $60 \mathrm{MPa}$. The creep rates vary inversely with an exponent of about 4.2 with inverse grain size. For Nabarro-Herring creep or for Coble creep according to Eq. (11) exponents of 2 or 3 would be expected. On the other hand, the authors of [6] assumed that all other factors in Eq. (11) are independent of grain size, which may not necessarily be a realistic possibility. 


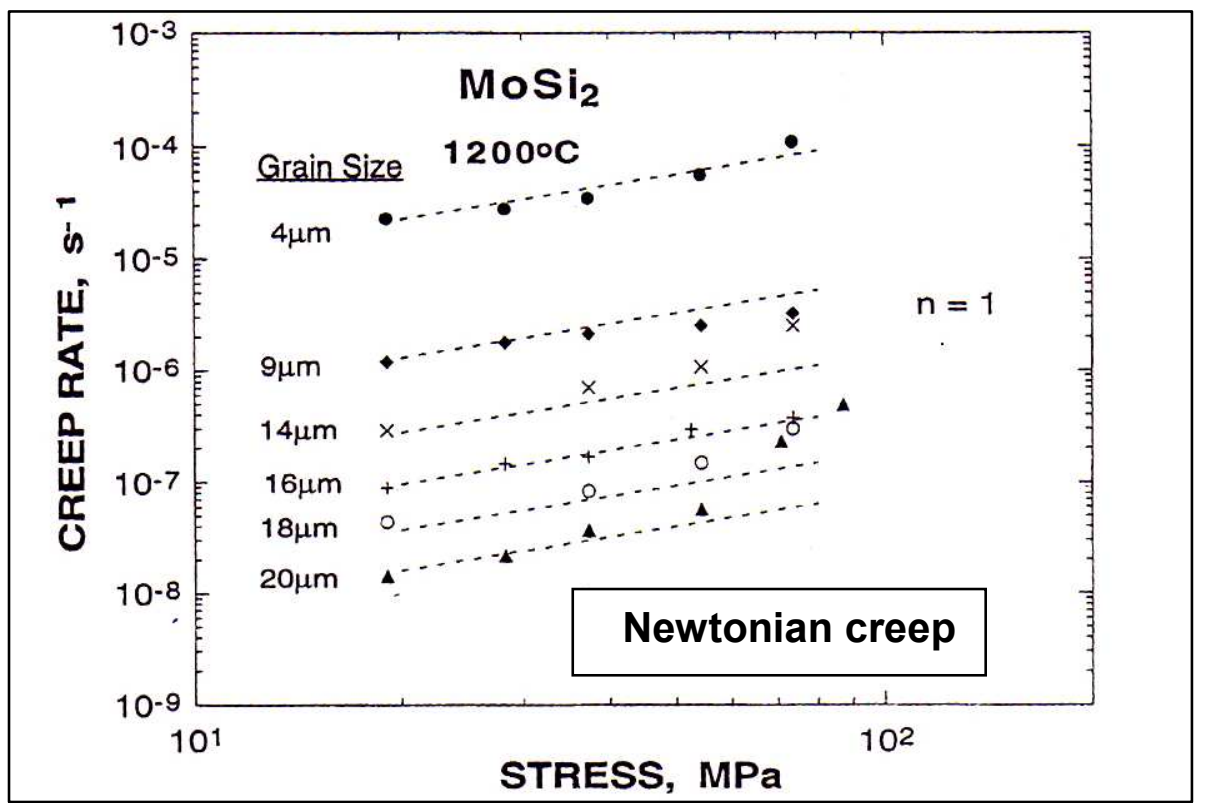

Figure 15 Effect of grain size on the creep rate of $\mathrm{MoSi}_{2}$ in the Newtonian range (diffusional creep) according to Sadananda et al. [6]. The dashed lines represent a stress exponent of $n=1$.

The effect of grain size on the creep rate in the power-law regime from the work of Sadananda et al. [6] is shown in Fig.16. Normally in the power-law creep regime, grain-size effects are rarely expected, since spacing between dislocations and not the grain size is the rate-controlling factor. Fig. 16 shows that for a decrease of grain size from 25 to $4 \mu \mathrm{m}$ the creep rate increases by three to four orders of magnitude. As indicted in Fig.16 the stress exponent $n$ is about 4 . The grain-size exponent (exponent of $1 / L$ ) in the power-law creep regime was found to be in the order of 4.3 to 4.4 similar to the grain-size exponent in the Newtonian regime [6]. In addition, it was also reported that the transition from the Newtonian to the power-law creep depends on the grain size and on the loadhistory.

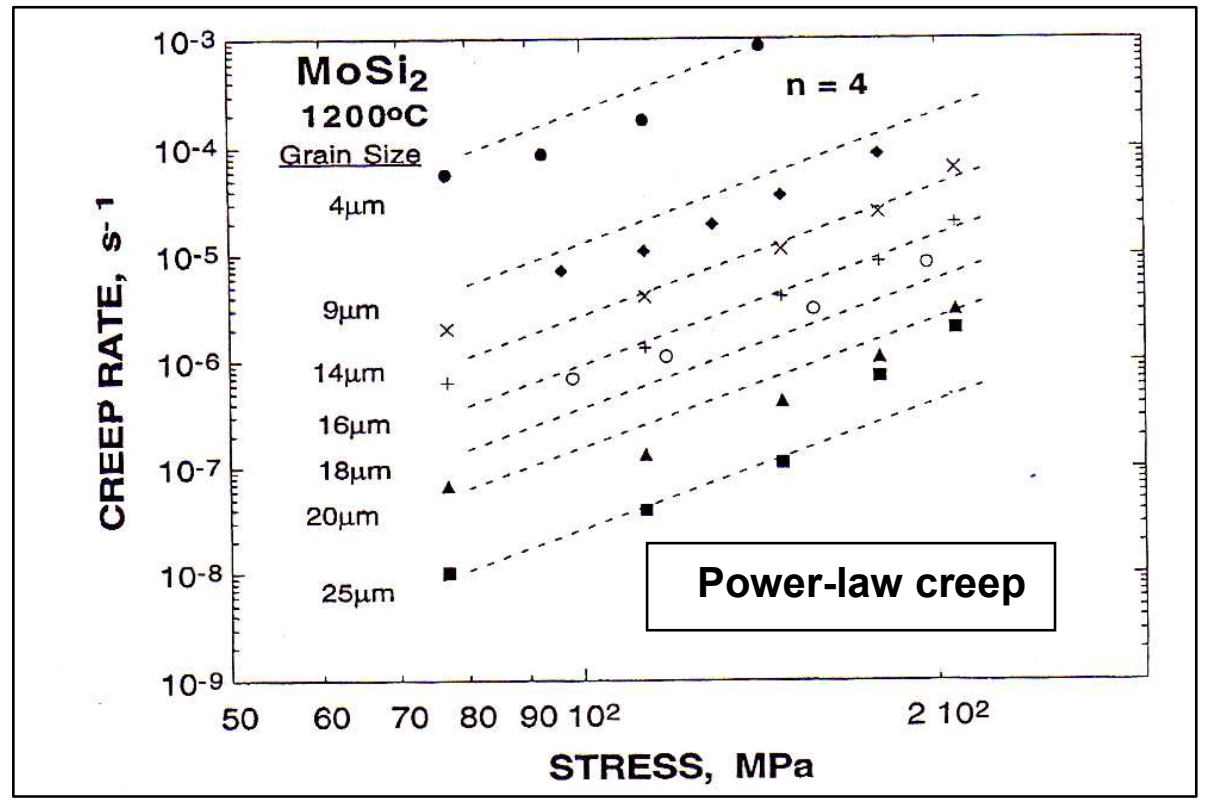

Figure 16 Effect of grain size on the creep rate of $\mathrm{MoSi}_{2}$ in the power law regime of creep according to Sadananda et al. [6]. The dashed lines represent a stress exponent of $n=4$. 
Sadananda et al. [6] also determined the activation enthalpy of Newtonian creep $(n=1)$ as a function of grain size after taking into account the dependence of elastic moduli of $\mathrm{MoSi}_{2}$ on temperature studied by Tanaka et al. [32]. Fig. 17 shows the activation enthalpy as a function of the grain size. It increases with increasing grain size and reaches a plateau value of about $430 \mathrm{~kJ} \mathrm{~mol}^{-1}$ for grain sizes above about $15 \mu \mathrm{m}$.

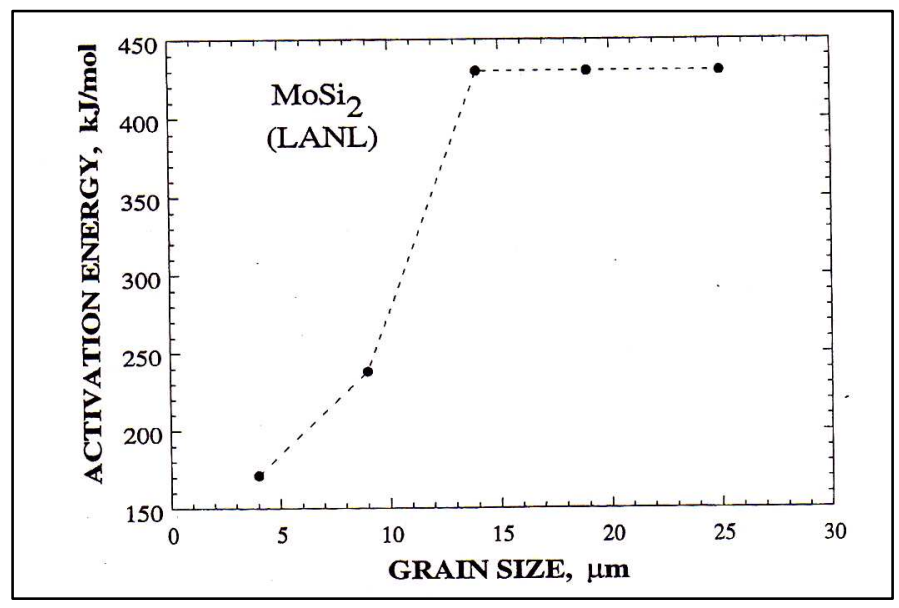

Figure 17 Activation enthalpy for Newtonian creep of $\mathrm{MoSi}_{2}$ as function of grain size according to Sadananda et al. [6].

Activation enthalpies for self-diffusion of $\mathrm{Mo}$ and $\mathrm{Si}$ in monocrystals of $\mathrm{MoSi}_{2}$ are available from the work of Salamon and Mehrer [11] discussed already the previous section (see also Fig. 3). The Arrhenius parameters of lattice diffusion of both components parallel and perpendicuelar to the tetragonal axis are listed in Table.1.

Table 1 Activation enthalpies $\boldsymbol{Q}$ and pre-exponential factors $\boldsymbol{D}^{\boldsymbol{\theta}}$ for the diffusion of radiotracers ${ }^{99} \mathrm{Mo}$ and ${ }^{31} \mathrm{Si}$ parallel and perpendicular to the tetragonal axis of molybdenum disilicide monocrystals according to Salamon and Mehrer [11].

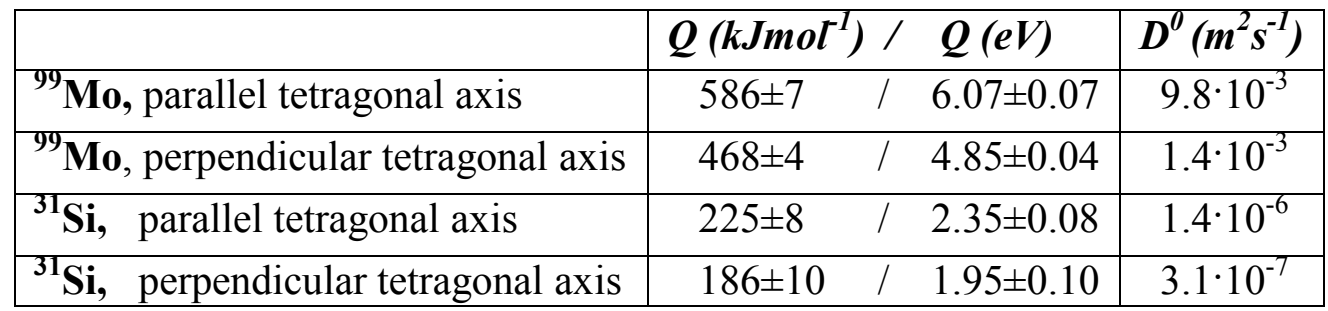

The activation enthalpy of Newtonian creep for grain sizes above about $15 \mu \mathrm{m}$ reaches 430 $\mathrm{kJmol}^{-1}$ or $4.45 \mathrm{eV}$ (see Fig. 17). Such a value is not far from the activation enthalpies of Mo diffusion of Table 1, which represents Mo lattice diffusion. For a stoichiometric line compound like $\mathrm{MoSi}_{2}$ diffusional creep via lattice diffusion requires long-range motion of both kinds of atoms. Although a theory of creep for stoichiometric binary compounds has not been formulated, the activation enthalpy of diffusional creep is expected to be a weighted average of the activation enthalpies of both types of atoms. The rate controlling process in $\mathrm{MoSi}_{2}$ according to the NernstPlanck equqtion very likely is the much slower diffusion of Mo.

For smaller grain sizes according to Fig. 17 the activation enthalpy of diffusional creep decreases significantly to about $170 \mathrm{kJmol}^{-1}$ or $1.76 \mathrm{eV}$. It is likely that such a value can be attributed to Coble creep, which implies grain-boundary diffusion. Neither data for grain-boundary nor dislocation-pipe diffusion in $\mathrm{MoSi}_{2}$ are available according to the authors' knowledge. 
Experience on grain-boundary diffusion in pure metals tells us that the activation enthalpies of grain-boundary diffusion lie between 40 to $70 \%$ of the activation enthalpies of lattice diffusion [30, 31]. We suggest that grain-boundary diffusion controls Newtonian creep for small grain sizes.

Dislocation climb in a binary ordered intermetallic such as $\mathrm{MoSi}_{2}$ also requires diffusion of both types of atoms. Transmission electron microscopy studies have shown that the dislocations contributing to creep are mainly edge dislocations in the basal planes of molybdenum disilicide [5]. Such dislocations are illustrated in Fig. 18. Their climb involves diffusion of two rows of Si and one row of Mo atoms. In the temperature range of the creep experiments diffusion of Mo is at least six orders of magnitude slower than diffusion of $\mathrm{Si}$, irrespective which diffusion direction is considered. The apparent activation enthalpy of creep (see, e.g. Fig. 14) lies between the activation enthalpies of lattice diffusion (see Table 1) of Mo and Si but closer to those of Mo. This suggests, that the much slower Mo diffusion is the rate-controlling factor of dislocation climb as well.

Although diffusion data for grain-boundary and dislocation pipe diffusion are not available it is very likely that the low mobility of Mo is also the rate-controlling factor in diffusion shortcircuits. Therefore, we conclude that it is mainly the kinetics of molybdenum diffusion that controls the creep behavior of molybdenum disilicide.

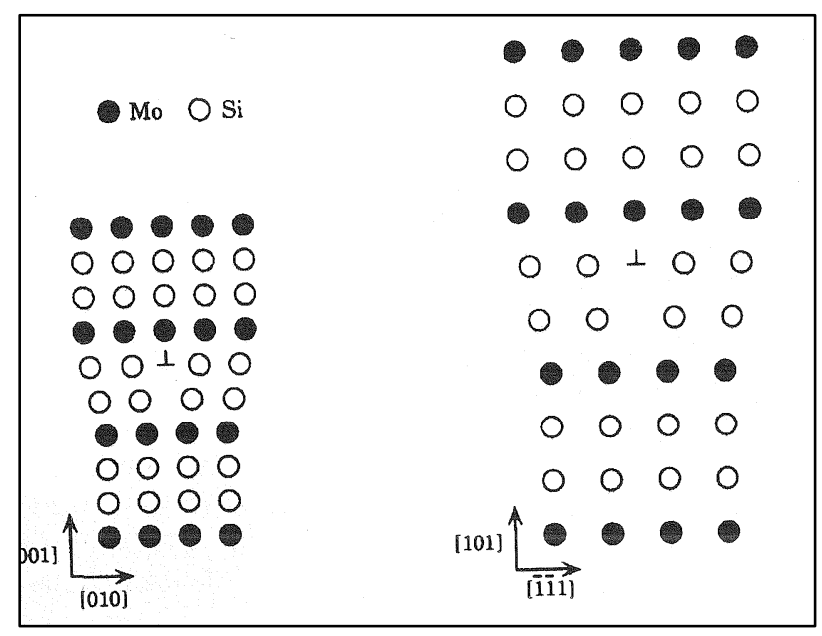

Figure 18 Schematic view of two types of edge dislocations in the basal plane of the molybdenum disilicide structure.

Unsolved problems: Several features of the experimentally observed creep behaviour [5, 6 . 29] cannot be explained in the framework of currently known theories of creep. We mention four of the deficiencies of available creep models, which have been developed mainly for pure metals but not for stoichiometric and uniaxial line compounds like $\mathrm{MoSi}_{2}$.

(i) It is not clear which type of diffusion coefficients - Mo or Si tracer diffusivities or interdiffusion coefficients according to Darken-Manning or Nernst.Planck should be used in the creep equations.

(ii) The reported dependence of Newtonian creep [6] on grain size does neither agree with the expected grain-size dependences of Nabarro-Herring nor with that of Coble creep.

(iii) The strong dependence of power-law creep on the grain size [6] also waits for explanation.

(iv) The orientation dependence of creep rates reported in [29] is not included in creep models. Although creep behaviour is usually studied by uniaxial stress, deformation takes place on a number of slip systems. In fine-grained polycrystalline samples this is a moot point as it is averaged out.

Finally we mention that grain-boundary diffusion data for molybdenum disilicide are missing. Such data would be very helpful for an improved understanding of high-temperature creep 


\section{Summary and Conclusions}

Self-diffusion of molybdenum and silicon and diffusion of germanium in both principal directions of tetragonal molybdenum disilicide [9-13] revealed the following major features:

- all diffusivities show Arrhenius behaviour;

- diffusion of molybdenum is orders of magnitude slower than diffusion of silicon and germanium;

- diffusion of silicon and germanium are relatively fast processes;

- diffusion of germanium is slightly faster than silicon diffusion, presumably due to an attractive interaction between germanium and vacancies in the Si sublattice;

- diffusion perpendicular to the tetragonal axis is for all three diffusants faster than diffusion parallel to the axis.

Positron annihilation studies [14] revealed the following facts about point defects in molybdenum disilicide:

- life-time studies show that thermal vacancies are formed with a formation enthalpy of $1.6 \mathrm{eV}$ or $154 \mathrm{~kJ} / \mathrm{mol}$.

- coincident Doppler broadening experiments have demonstrated that Si sublattice vacancies dominate the point defect population in molybdenum disilicide up to the highest temperature studied in the positron experiments (1343 K).

Taken together, knowledge about diffusion and vacancy properties of $\mathrm{MoSi}_{2}$ suggests that diffusion of Si and Mo occurs via vacancies on the relevant sublattices. Vacancy-mediated diffusion is a correlated process and the expressions for the diffusivities in both principal directions and for both components contain correlation factors:

- correlation factors for Si diffusion are available from calculations of two different groups [16, 17], the results of which are in good agreement. The temperature dependence of the correlation factor contributes parallel to the tetragonal axis contributes $39 \mathrm{~kJ} \mathrm{~mol}^{-1}$ or $0.4 \mathrm{eV}$ to the activation enthalpy of Si diffusion

- correlation factors for Mo diffusion have been deduced recently by Monte Carlo simulations [17]. In the temperature range of the diffusion experiments the correlation factors of Mo diffusion are constants and hence do not contribute to the activation enthalpies of Mo diffusion.

Creep experiments on polycrystals from literature $[5,6]$ indicate that the creep mode is a transition between dislocation creep and diffusion creep. In large-grain polycrystals, Newtonian creep occurs via lattice diffusion, which is rate-controlled according to the Nernst-Planck interdiffusin coefficient by the slow Mo diffusion. For smaller grain size grain-boundary diffusion is presumably the process that controls Newtonian creep. Steady-state creep in the power-law regime occurs via dislocation climb, which also requires diffusion of both silicon and molybdenum. In general, it is mainly the slow diffusion of molybdenum that controls the creep behavior of molybdenum disilicide.

Several features of the creep behaviour in the stoichiometric line compound molybdenum disilicide, including the strong grain-size dependence of power-law creep, cannot be explained by available theories of creep. Creep models have been developed for pure metals only and not for binary intermetallics. It is, for example, not clear which of the several diffusivities of a binary compound should enter the equations of power-law, Nabarro.Herring or Coble creep. Our suggestion without prove is to use the Nernst-Planck interdiffusion coefficient. Grain-boundary diffusion data of molybdenum silicide are missing and could help to improve the understanding of the creep behaviour. 


\section{Acknowledgements.}

One of the authors (H.M) is grateful to Profs. Irina Belova and Graeme Murch for the invitation to join the 'Centre of Mass and Thermal Transport in Engineering Materials' at The University of Newcastle, Australia, as visiting professor during October to December 2010. He also expresses thanks to all members of the Centre for their continuous help and their warm hospitality. We are grateful to Prof. W. Sprengel from the Graz University of Technology (Austria) for comments on the manuscript.

\section{References}

[1] O. Hoenigschmidt: Monatshefte Chemie Vol. 28 (1907), p. 1017.

[2] Kanthal: Swedish Patent Vol. 155 (1953), p. 836.

[3] A.K. Vasudevan, J.J. Petrovic: Mat. Sci. and Eng. Vol. A155 (1992), pp.1-17.

[4] Z. Yao, J.J. Stiglich, T.S. Sudarshan: Materials Modification, Inc., Fairfax, VA 22031; and J. of Materials Engineering and Performances Vol. 8 (1999), pp. 291 - 304.

[5] K. Sadananda, C.R. Feng, H. Jones: Mat. Sci. and Eng. Vol. A155 (1992), pp. 227 - 239.

[6] K. Sadananda, C.R. Feng, R. Mitra, S.C. Deevi: Mat. Sci. and Eng. Vol. A261 (1999), pp. 223 -238 .

[7] J.H. Westbrook, R.L. Fleischer (Eds.), Intermetallic Compounds, Principles and Practice, Vol.3 - Progress, Chapter 16, Creep (by D.Shah and E.Lee), J.Wiley and Sons, Chichester, 2002.

[8] W.J. Boettiger, J.H. Perepezko, P.S. Frankwicz: Mat. Sci. and Eng. Vol. A155 (1992), p. 33.

[9] M. Salamon, H. Mehrer: Defect and Diffusion Forum Vols. 216 - 217 (2003), pp. 161 - 168.

[10] M. Salamon, A. Strohm, T. Voss, P. Laiinen, I. Rihimaeki, S. Divinski, W. Frank, J. Raeisaenen, H. Mehrer: Philos. Mag. Vol. 84 (2004), pp. 737- 756.

[11] M. Salamon, H. Mehrer: Z. Metallkd. Vol. 8 (2005), pp. 833-841.

[12] M. Salamon, PhD thesis, Universität Münster; Germany, 2003.

[13] H. Mehrer: Defect and Diffusion Forum Vols. 297- 301 (2010), p. 1377.

[14] Y. Zhang, W. Sprengel, T.E.M. Staab, H. Inui, H.-E. Schaefer: Phys. Rev. Letters Vol. 92 (2004), p. 155502.

[15] H.-E. Schaefer, Nanoscience, Springer, Heidelberg, Dordrecht, London, New York, 2010.

[16] S.V. Divinski, M. Salamon, H. Mehrer: Phil. Mag. Vol. 84 (2004), pp. 757-772.

[17] I.V. Belova, H. Mehrer, G.E. Murch: Phil. Mag., in print.

[18] J. Bardeen, C. Herring, in: Atom Movements, A.S.M. Cleveland, 1951, p. 87.

[19] R.E. Howard: Phys. Rev. Vol. 144 (1966), p. 650.

[20] J.R. Manning, Diffusion Kinetics for Atoms in Crystals, Princeton, New Jersey, van Norstrand, 1968.

[21] A.D. LeClaire, Physical Chemistry -- an Advanced Treatise, Vol. X, Chapter 5, Academic Press, New York, 1970.

[22] G.E. Murch, Simulation of the Diffusion Kinetics with the Monte Carlo Method, in: G.E. Murch and A.S. Nowick (Eds.), Diffusion in Crystalline Solids, Academic Press, 1984, pp.379-427.

[23] H.-E. Schaefer: Phys. Stat. Sol. (a) Vol. 102 (1987), p. 47. 
[24] H.-E. Schaefer, F. Baier, M.A. Müller, K.J. Reichle, K. Reimann, A.A. Rempel, K. Sato, F. Ye, X.Y. Zhang, W. Sprengel: Phys. Stat. Sol. (b), in print.

[25] M.F. Ashby, D.R.H. Jones, Engineering Materials -- An Introduction to their Properties and Application, Pergamon Press, 1980.

[26] J. Philibert, Atom Movements -- Diffusion and Mass Transport in Solids, Les Editions de Physique, Les Ulis, Cedex A, France, 1991.

[27] P. Shewmon, Diffusion in Solids, The Minerals, Metals and Materials Society, Warrendale, Pennsylvania, 1989.

[28] H. Schaumburg, Werkstoffe, B.G. Teubner, Stuttgart, 1990.

[29] H. Inui, K. Ishikawa, M. Yamaguchi: Intermetallics Vol. 8 (2000), pp. 1159-1168.

[30] H. Mehrer (Vol. Ed.), Diffusion in Solid Metals and Alloys, Landolt-Börnstein, New Series, Group III, Vol. 26, Springer-Verlag, 1990.

[31] H. Mehrer, Diffusion in Solids - Fundamentals, Methods, Materials, Diffusion-Controlled Processes, Springer Series in Solid State Science 155, 2007.

[32] K. Tanaka, H. Onome, H. Inui, M. Yamaguchi, M. Koiwa: Mat. Sci. and Eng. Vol. A239-240 (1997), p. 188.

[33] K. Matsuda, Y. Shirai, M. Yamaguchi: Intermetallics Vol. 6 (1997), pp. 395-401. 


\section{Recent Advances in Mass Transport in Materials}

10.4028/www.scientific.net/DDF.322

\section{Molybdenum Disilicide - Diffusion, Defects, Diffusion Correlation, and Creep}

10.4028/www.scientific.net/DDF.322.107

\section{DOI References}

[1] O. Hoenigschmidt: Monatshefte Chemie Vol. 28 (1907), p.1017.

http://dx.doi.org/10.1007/BF01512021

[4] Z. Yao, J.J. Stiglich, T.S. Sudarshan: Materials Modification, Inc., Fairfax, VA 22031; and J. of Materials Engineering and Performances Vol. 8 (1999), pp.291-304.

doi:10.1361/105994999770346837

[5] K. Sadananda, C.R. Feng, H. Jones: Mat. Sci. and Eng. Vol. A155 (1992), p.227 - 239.

doi:10.1016/0921-5093(92)90329-Y

[6] K. Sadananda, C.R. Feng, R. Mitra, S.C. Deevi: Mat. Sci. and Eng. Vol. A261 (1999), p.223 - 238.

doi:10.1016/S0921-5093(98)01070-3

[7] J.H. Westbrook, R.L. Fleischer (Eds. ), Intermetallic Compounds, Principles and Practice, Vol. 3 Progress, Chapter 16, Creep (by D. Shah and E. Lee), J. Wiley and Sons, Chichester, (2002).

doi:10.1002/0470845856.ch16

[9] M. Salamon, H. Mehrer: Defect and Diffusion Forum Vols. 216 - 217 (2003), p.161 - 168.

doi:10.4028/www.scientific.net/DDF.216-217.161

[10] M. Salamon, A. Strohm, T. Voss, P. Laiinen, I. Rihimaeki, S. Divinski, W. Frank, J. Raeisaenen, H. Mehrer: Philos. Mag. Vol. 84 (2004), pp.737-756.

doi:10.1080/14786430310001641966

[13] H. Mehrer: Defect and Diffusion Forum Vols. $297-301$ (2010), p.1377.

doi:10.4028/www.scientific.net/DDF.297-301.1377

[14] Y. Zhang, W. Sprengel, T.E.M. Staab, H. Inui, H. -E. Schaefer: Phys. Rev. Letters Vol. 92 (2004), p.155502.

doi:10.1103/PhysRevLett.92.155502

[16] S.V. Divinski, M. Salamon, H. Mehrer: Phil. Mag. Vol. 84 (2004), pp.757-772.

doi:10.1080/14786430310001646781

[19] R.E. Howard: Phys. Rev. Vol. 144 (1966), p.650.

http://dx.doi.org/10.1103/PhysRev.144.650

[23] H. -E. Schaefer: Phys. Stat. Sol. (a) Vol. 102 (1987), p.47.

http://dx.doi.org/10.1002/pssa.2211020104

[29] H. Inui, K. Ishikawa, M. Yamaguchi: Intermetallics Vol. 8 (2000), pp.1159-1168.

doi:10.1016/S0966-9795(00)00055-8

[30] H. Mehrer (Vol. Ed. ), Diffusion in Solid Metals and Alloys, Landolt-Börnstein, New Series, Group III, Vol. 26, Springer-Verlag, (1990).

doi:10.1007/10390457 26

[32] K. Tanaka, H. Onome, H. Inui, M. Yamaguchi, M. Koiwa: Mat. Sci. and Eng. Vol. A239-240 (1997), p.188.

doi:10.1016/S0921-5093(97)00580-7 
[33] K. Matsuda, Y. Shirai, M. Yamaguchi: Intermetallics Vol. 6 (1997), pp.395-401. http://dx.doi.org/10.1016/S0966-9795(97)00082-4 